University of Nebraska - Lincoln

DigitalCommons@University of Nebraska - Lincoln

Ralph Skomski Publications

Research Papers in Physics and Astronomy

December 1993

\title{
Giant energy product in nanostructured two-phase magnets
}

Ralph Skomski

University of Nebraska-Lincoln, rskomski2@unl.edu

J.M.D. Coey

Trinity College, Dublin, Ireland

Follow this and additional works at: https://digitalcommons.unl.edu/physicsskomski

Part of the Physics Commons

Skomski, Ralph and Coey, J.M.D., "Giant energy product in nanostructured two-phase magnets " (1993). Ralph Skomski Publications. 32.

https://digitalcommons.unl.edu/physicsskomski/32

This Article is brought to you for free and open access by the Research Papers in Physics and Astronomy at DigitalCommons@University of Nebraska - Lincoln. It has been accepted for inclusion in Ralph Skomski Publications by an authorized administrator of DigitalCommons@University of Nebraska - Lincoln. 


\title{
Giant energy product in nanostructured two-phase magnets
}

\author{
Ralph Skomski and J. M. D. Coey \\ Department of Pure and Applied Physics, Trinity College, Dublin 2, Ireland
}

(Received 25 August 1993)

\begin{abstract}
Exchange hardening of nanostructured two-phase systems composed of an aligned hard phase and a soft phase with high magnetization is investigated using an approach which yields analytic nucleation fields from the micromagnetic vector equation, and accounts for interactions between the soft regions. In suitable structures the nucleation field is proportional to the volume-averaged anisotropy constant. For example, a multilayer composed of alternating $2.4 \mathrm{~nm}$ hard-magnetic $\mathrm{Sm}_{2} \mathrm{Fe}_{17} \mathrm{~N}_{3}$ layers and $9 \mathrm{~nm}$ $\mathrm{Fe}_{65} \mathrm{Co}_{35}$ layers can have an energy product as high as $1 \mathrm{MJ} / \mathrm{m}^{3}(120 \mathrm{MGOe})$, with a rare-earth content of only $5 \mathrm{wt} \%$. Giant energy products may also be achieved in suitable cellular and disordered structures.
\end{abstract}

A key characteristic of a permanent magnet of volume $V$ is the energy product $(B H)_{\max }=(1 / V) \int_{\text {magnet }}|\mathbf{B} \cdot \mathbf{H}| d \mathbf{r}$ which is twice the maximum magnetostatic energy available from a magnet of optimal shape. Energy product increases with coercivity $H_{c}$ and remanence $M_{r}$ but it can never exceed the value $\mu_{0} M_{r}^{2} / 4$ corresponding to an ideal rectangular hysteresis loop. An upper limit for the remanence is the spontaneous magnetization $M_{0}$ but if magnetization were the only consideration then $\alpha$-iron with $\mu_{0} M_{0}=2.15 \mathrm{~T}$ would be used for permanent magnets with energy products as high as $920 \mathrm{~kJ} / \mathrm{m}^{3}$. In fact, the coercivity of bcc iron is so low that energy products of iron magnets are only of order $1 \mathrm{~kJ} / \mathrm{m}^{3}$. In the past it was necessary to resort to cumbersome bar and horseshoe shapes to avoid spontaneous demagnetization into a multidomain state by the magnet's own magnetostatic field.

Modern high-performance magnets ${ }^{1}$ such as $\mathrm{Nd}_{2} \mathrm{Fe}_{14} \mathrm{~B}$ or $\mathrm{Sm}_{2} \mathrm{Fe}_{17} \mathrm{~N}_{3}$ overcome this problem by exchangecoupling iron atoms to rare-earth atoms in sites with strong uniaxial anisotropy. The rare-earth sublattice yields anisotropy fields which exceed the magnetostatic fields by an order of magnitude. The penalty, however, is a reduced magnetization due to the rare-earth and nonmagnetic elements. The light rare-earths' atomic moments are, at best, slightly larger than that of iron, but they occupy more than three times the volume. Nevertheless, it has been possible to use $\mathrm{Nd}_{2} \mathrm{Fe}_{14} \mathrm{~B}$, which has $\mu_{0} M_{0}=1.61 \mathrm{~T}$ and $\mu_{0} M_{0}^{2} / 4=516 \mathrm{~kJ} / \mathrm{m}^{3}$ to achieve energy products as high as $405 \mathrm{~kJ} / \mathrm{m}^{3}$ in laboratory-scale magnets. ${ }^{2}$

The outlook for discovering new ternary phases with magnetization significantly higher than that of those available at present is poor. Interstitial modification with small atoms such as nitrogen or carbon is effective for enhancing Curie temperature and anisotropy, but the magnetization remains practically unchanged. ${ }^{3,4} \mathrm{~A}$ different approach is necessary if further progress is to be possible.

Recently, magnetic hardening has been achieved in the nanocrystalline composites $\mathrm{Nd}_{2} \mathrm{Fe}_{14} \mathrm{~B} / \mathrm{Fe}_{3} \mathrm{~B}$ and $\mathrm{Sm}_{2} \mathrm{Fe}_{17} \mathrm{~N}_{3} / \mathrm{Fe}$ produced by melt spinning ${ }^{5}$ and mechani- cal alloying, ${ }^{6}$ respectively. The idea behind these systems is that the comparatively low remanance $M_{r} \approx M_{0} / 2$ of the isotropic hard phase can be improved by exchange coupling with a soft phase..$^{5-10}$ But the energy product, though improved with respect to the isotropic singlephase rare-earth material, does not reach the level attained in oriented rare-earth magnets.

Here we show how it should be possible to substantially increase the energy product in oriented nanostructured two-phase magnets by exploiting exchange coupling between hard and soft regions. We derive analytic results for the nucleation field and energy product, and suggest how the composites might best be structured.

To describe the magnetic reversal, we consider the free energy ${ }^{11,12}$

$F=\int\left[A(\mathbf{r})\left[\frac{\nabla \mathbf{M}}{M_{0}}\right]^{2}-K_{1}(\mathbf{r}) \frac{(\mathbf{M} \cdot \mathbf{n})^{2}}{M_{0}^{2}}-\mu_{0} \mathbf{M} \cdot \mathbf{H}\right] d \mathbf{r}$,

where $H$ denotes the internal field, which is the sum of the externally applied field and the magnetostatic "demagnetizing" field. $A(\mathbf{r})$ is the exchange stiffness, $M(\mathbf{r})$ with $|M(\mathbf{r})|=M_{0}$ is the local magnetization, and $K_{1}(\mathbf{r})$ is the first anisotropy constant. The unit vector in the easy-axis direction $\mathbf{n}$ is assumed not to depend on $\mathbf{r}$ (common $c$ axis). ${ }^{13}$ The hard and soft phases can have different values of the parameters $A, M_{0}$, and $K_{1}$ denoted by the suffixes $h$ and $s$. Note that the ferromagnetic exchange interaction $[A(\mathbf{r})>0]$ in iron-rich intermetallics is dominated by the iron sublattice (cf. Ref. 3); we do not consider nonmagnetic regions which are able to cause oscillating coupling effects such as those observed in multilayers with giant magnetoresistance.

We start from the perfectly aligned state where $M(\mathbf{r})=M_{0} \mathbf{n}$. If a sufficiently high internal field is reached, the aligned state becomes unstable, and magnetic reversal begins (nucleation). Nucleation is a necessary but not sufficient condition for complete magnetic reversal. It sets a lower limit to the coercive field, $H_{c} \geq H_{N}$, since there is a possibility that the reversed nucleus will 
not propagate. ${ }^{14-16}$ The energy product can be estimated from the hysteresis loop by putting $H_{c}=H_{N}$.

In special cases, the nucleation problem [Eq. (1)] has been solved in one, two, or three dimensions by series expansion or an appropriate ansatz. ${ }^{12,14,17-19}$ Here we take the vector identity,

$M(\mathbf{r})=M_{0}(\mathbf{r})\left(m_{x}(\mathbf{r}) \mathbf{e}_{x}+m_{y}(\mathbf{r}) \mathbf{e}_{y}+\sqrt{1-m_{x}^{2}-m_{y}^{2}} \mathbf{e}_{z}\right)$,

and expand the free-energy density with respect to the small transverse components $m_{x}, m_{y} \ll 1,{ }^{20}$ and minimize the free energy to yield the micromagnetic equation,

$$
\nabla \Psi(A(\mathbf{r}) \nabla \mathbf{m})-K_{1}(\mathbf{r}) \mathbf{m}+\frac{1}{2} \mu_{0} M_{0}(\mathbf{r}) H_{N} \mathbf{m}=0,
$$

with $\mathbf{m}=m_{x}(\mathbf{r}) \mathbf{e}_{x}+m_{y}(\mathbf{r}) \mathbf{e}_{y}$. Note that $\mathbf{n}=\mathbf{e}_{z}$ does not enter the micromagnetic vector equation, so long as $\mathbf{H}$ is applied in the $-\mathbf{n}$ direction (cf. Ref. 11). This equation represents an eigenvalue problem, and to find $H_{N}$ it is sufficient to solve the differential equation,

$$
-\frac{2 A(\mathbf{r})}{\mu_{0} M_{0}(\mathbf{r})} \nabla^{2} \Psi+\frac{2 K_{1}(\mathbf{r})}{\mu_{0} M_{0}(\mathbf{r})} \Psi=H_{N} \Psi,
$$

with the interface boundary condition $A_{s}(\mathrm{e} \cdot \nabla) \Psi_{s}$ $=A_{h}(\mathbf{e} \cdot \nabla) \Psi_{h}$. Here $\Psi$ represents $m_{x}$ or $m_{y}$ or any linear combination of the two, and $e$ is the unit vector normal to the interface. The boundary condition reflects the fact that phases with strong exchange stiffness tend to realize a more homogeneous magnetization state $(\nabla \Psi$ and $\nabla \mathrm{m}$ small). Apart from a minor generalization of the boundary condition, Eq. (4) corresponds to Schrödinger's equation for a particle moving in a three-dimensional potential $2 K_{1}(\mathbf{r}) / \mu_{0} M_{0}(\mathbf{r})$, which allows us to apply ideas familiar from quantum mechanics to discuss micromagnetics. In particular, the nucleation field corresponds to the quantum-mechanical ground-state energy, and the small transverse magnetization or nucleation mode $\Psi$ has its analog in the wave function.

First we consider an ideally soft inclusion (diameter $D$, $K_{s}=0$ ) in a hard matrix (Fig. 1). The initial state is one where the magnetization of the matrix and inclusion are perfectly aligned. Introducing spherical coordinates and taking the limit $\Psi \rightarrow 0$ as $r \rightarrow \infty$ we obtain the eigenvalue

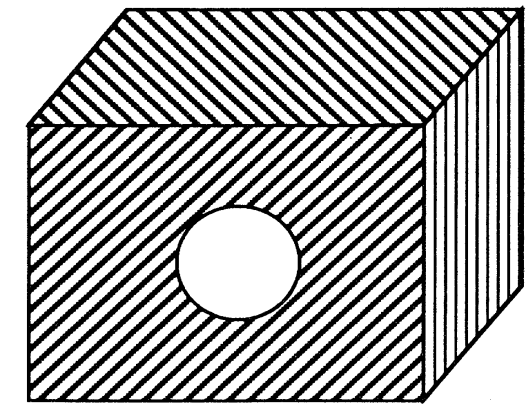

FIG. 1. Spherical soft inclusion with diameter $D$ in a hardmagnetic matrix (dashed region). equation,

$$
\begin{gathered}
\frac{A_{s}}{A_{h}}\left[\frac{D}{2} \sqrt{\mu_{0} M_{s} H_{N} / 2 A_{s}} \cot \left[\frac{D}{2} \sqrt{\mu_{0} M_{s} H_{N} / 2 A_{s}}\right]-1\right] \\
+1+\frac{D}{2} \sqrt{\left(2 K_{h}-\mu_{0} M_{h} H_{N}\right) / 2 A_{h}}=0
\end{gathered}
$$

which can be solved numerically (Fig. 2). The nucleation field reaches a plateau below $D \approx \delta_{h}$, where $\delta_{h}$ is the Bloch wall width of the hard phase.

Taking values appropriate to $\mathrm{Sm}_{2} \mathrm{Fe}_{17} \mathrm{~N}_{3}$, the intermetallic with the most favorable combination of magnetization and anisotropy, ${ }^{3,21}$ inclusions smaller than $3 \mathrm{~nm}$ in diameter will have coercivity equal to the anisotropy field of the matrix $(\approx 20 \mathrm{~T})$. Even at a diameter $D=7 \mathrm{~nm}$, the soft inclusion retains a coercivity $\mu_{0} H_{N}=7 \mathrm{~T}$. For larger inclusions the coercivity falls off as $1 / D^{2}$; inhomogeneous magnetostatic fields can initiate nucleation at diameters greater than $20 \mathrm{~nm}$.

For sufficiently small reverse fields $|H|<H_{N}(D)$, the single soft inclusion is ideally aligned along $e_{z}$ and slightly enhances the remanence, provided $M_{s}>M_{h}$. To obtain a significant remanence enhancement it is necessary to generalize to large numbers of spherical inclusions per unit volume (Fig. 3). The remanence is

$$
M_{r}=f_{h} M_{h}+f_{s} M_{s},
$$

where $f_{s}$ and $f_{h}=\left(1-f_{s}\right)$ are the volume fractions of the soft and hard phases, respectively, but when the distance between neighboring inclusions becomes too small, the soft regions interact and coercivity is destroyed. This behavior is illustrated schematically in Fig. 4. In the case of well-separated inclusions, the lowest-lying magnetization mode which is responsible for nucleation decreases exponentially in the hard region. But when the distance between the soft inclusions is small, the magnetization modes can tunnel through the hard region, which no longer acts as an effective potential barrier. In fact, this micromagnetic exchange interaction can reduce the nu-

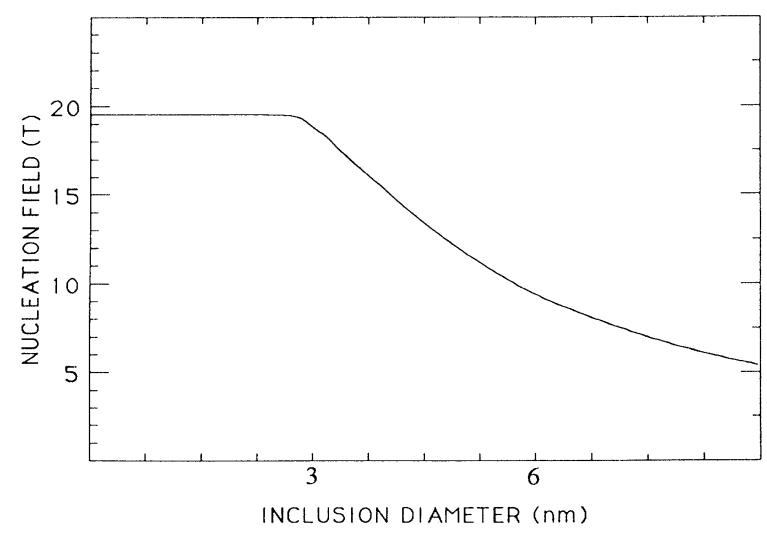

FIG. 2. Nucleation field $H_{N}$ as a function of $D$, the diameter of the inclusion in Fig. 1. The values assumed are for the $\mathrm{Sm}_{2} \mathrm{Fe}_{17} \mathrm{~N}_{3} / \mathrm{Fe}$ system: $\mu_{0} M_{s}=2.15 \quad \mathrm{~T}, \quad \mu_{0} M_{h}=1.55 \quad \mathrm{~T}$, $A_{s} / A_{h}=1.5, K_{s}=0$, and $K_{h}=12 \mathrm{~kJ} / \mathrm{m}^{3}$. 


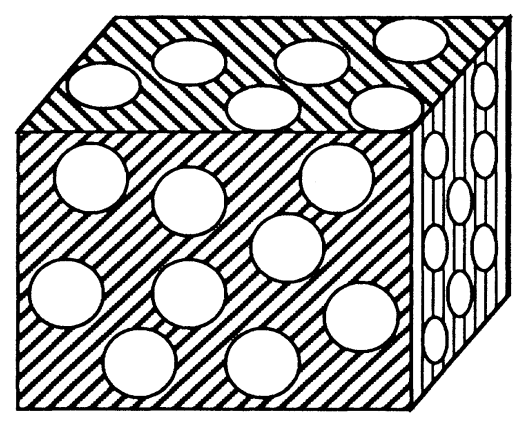

FIG. 3. A possible structure of remanence enhancement, with many inclusions per unit volume.

cleation field considerably when the thickness of the hard region is less than $\delta_{h}$.

In the plateau region (Fig. 2), where the soft regions are very small, the problem can be treated in perturbation theory. ${ }^{22}$ As in quantum mechanics, the lowest-order eigenvalue correction is obtained by using the normalized unperturbed function $\Psi_{0}$, so an effective anisotropy constant $K_{\text {eff }}$,

$$
\begin{aligned}
K_{\mathrm{eff}} & =\int \Psi^{*}(\mathbf{r}) K_{1}(\mathbf{r}) \Psi(\mathbf{r}) d \mathbf{r}, \\
& =\left\langle K_{1}(\mathbf{r})\right\rangle=f_{s} K_{s}+f_{h} K_{h},
\end{aligned}
$$

is introduced. The nucleation field is given by

$$
H_{N}=2\left\langle K_{1}(\mathbf{r})\right\rangle / \mu_{c}\left\langle M_{0}(\mathbf{r})\right\rangle
$$

or
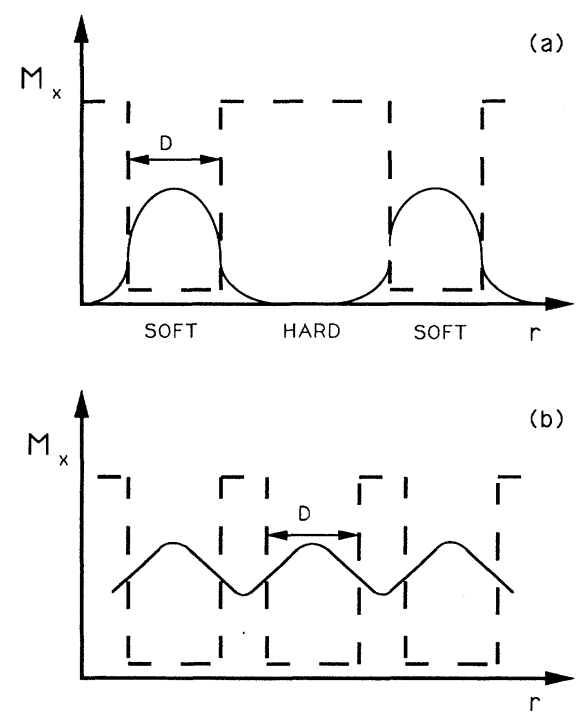

FIG. 4. Schematic diagram showing the effect of interaction between different soft regions. (a) The distance between the inclusions is large, and the magnetization node decreases exponentially in the hard region. (b) The distance between the inclusions is small, and micromagnetic exchange reduces the coercivity.

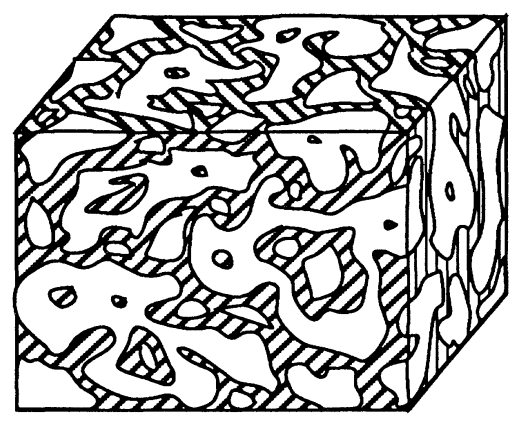

FIG. 5. An oriented, disordered two-phase magnet with common $c$ axis. The size of the soft and hard regions is smaller than the Bloch wall thickness $\delta_{h}$ of the hard phase.

$$
\mu_{0} H_{N}=2 \frac{f_{s} K_{s}+f_{h} K_{h}}{f_{s} M_{s}+f_{h} M_{h}} .
$$

If we neglect further pinning of the nucleus, we obtain a rectangular hysteresis loop with $H_{c}=H_{N}$ and $M_{r}=\left\langle M_{0}(\mathbf{r})\right\rangle$. The energy product depends on the shape of the magnet, but the optimal value is given by $\mu_{0} M_{r}^{2} / 4$ for $H_{N}>M_{r} / 2$ and $\mu_{0} H_{N} M_{r} / 2$ for $H_{N}<M_{r} / 2$. Using Eqs. (6) and (8) we find the highest-energy product obtained for $H_{N}=M_{r} / 2$. Putting $K_{s}=0$ yields

$$
(B H)_{\max }=\frac{1}{4} \mu_{0} M_{s}^{2}\left[1-\frac{\mu_{0}\left(M_{s}-M_{h}\right) M_{s}}{2 K_{h}}\right] .
$$

Due to the large $K_{h}$, the second term in the parentheses is small so the energy product approaches the ultimate value of $\mu_{0} M_{s}^{2}$. The corresponding volume fraction of the hard phase is

$$
f_{h}=\frac{\mu_{0} M_{s}^{2}}{4 K_{h}} \text {. }
$$

If we consider the $\mathrm{Sm}_{2} \mathrm{Fe}_{17} \mathrm{~N}_{3} / \mathrm{Fe}$ system and take values $\mu_{0} M_{s}=2.15 \mathrm{~T}, \mu_{0} M_{h}=1.55 \mathrm{~T}$, and $K_{h}=12$ $\mathrm{MJ} / \mathrm{m}^{3}$, we obtain a theoretical energy product of 880 $\mathrm{kJ} / \mathrm{m}^{3}$ (110 MG Oe) for a volume fraction of only $7 \%$ of

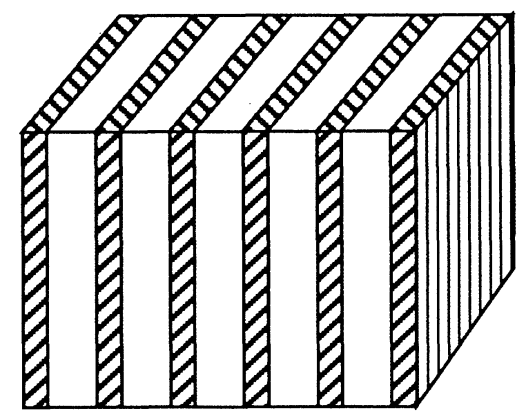

FIG. 6. A multilayer structure composed of alternating hardand soft- magnetic regions. The multilayer periodicity should not exceed $\sim 10 \mathrm{~nm}$. 
the hard phase. A further increase of the energy product is possible if iron is replaced by $\mathrm{Fe}_{65} \mathrm{Co}_{35}$ with $\mu_{0} M_{0}=2.43 \mathrm{~T}$; the theoretical energy product of the $\mathrm{Sm}_{2} \mathrm{Fe}_{17} \mathrm{~N}_{3} / \mathrm{Fe}_{65} \mathrm{Co}_{35}$ system might be as high as 1090 $\mathrm{kJ} / \mathrm{m}^{3}$ (137 MG Oe), with $f_{h}=9 \%$. It is remarkable that these optimum magnets are composed nearly entirely of $3 d$ metals, with only about 2 wt \% samarium.

The nucleation field [Eq. (8)] is independent of the shape of the soft regions, so long as their size lies in the plateau region (Fig. 2). The hard regions act as a skeleton to stiffen the magnetization direction of the soft regions. The practical problem, however, is to realize a structure where the soft regions are sufficiently small to avoid nucleation at small fields while having the hard regions crystallographically oriented. One conceivable solution is a disordered two-phase magnet (Fig. 5) with a common $c$ axis throughout the hard regions, but it is difficult to see how this might be achieved in practice. A more realistic possibility may be a multilayered structure of alternating soft and hard-magnetic layers (Fig. 6). The micromagnetic multilayer problem is analogous to the periodic multiple quantum-well problem; assuming a uniform demagnetizing field the solution for the lowest eigenvalue $H_{N}$ is given by the implicit equation,

$\sqrt{\left(2 K_{h}-\mu_{0} M_{h} H_{N}\right) / 2 A_{h}} \tanh \left(\frac{\lambda_{h}}{2} \sqrt{\left(2 K_{h}-\mu_{0} M_{h} H_{N}\right) / 2 A_{h}}\right)=\frac{A_{s}}{A_{h}} \sqrt{\mu_{0} M_{s} H_{N} / 2 A_{s}} \tan \left(\frac{\lambda_{s}}{2} \sqrt{\mu_{0} M_{s} H_{N} / 2 A_{s}}\right)$,

where $\lambda_{h}$ and $\lambda_{s}$ denote the thickness of the hard and soft layers, respectively. Equation (11) permits direct numerical calculation of the critical thickness $\lambda_{c}$ of the soft layer above which nucleation occurs, as a function of $\lambda_{h}$ and the required coercivity $H_{N}$. For example, to obtain a megajoule magnet using $\mathrm{Sm}_{2} \mathrm{Fe}_{17} \mathrm{~N}_{3}$, we need a coercivity of $1.12 \mathrm{~T}$ with a remanence of $2.24 \mathrm{~T}$ which corresponds to $79 \mathrm{vol} \% \mathrm{Fe}_{65} \mathrm{Co}_{35}$, thus fixing the ratio $\lambda_{s} / \lambda_{h}$. Taking $A_{s}=1.67 \times 10^{-11} \mathrm{~J} / \mathrm{m}$ and $A_{h}=1.07 \times 10^{-11} \mathrm{~J} / \mathrm{m}$, we obtain the values $\lambda_{h}=2.4 \mathrm{~nm}$ and $\lambda_{c}=\lambda_{s}=9.0 \mathrm{~nm}$. To achieve the same coercivity when $\lambda_{h} \rightarrow \infty$ we find $\lambda_{c}=9.5 \mathrm{~nm}$, the difference being due to the micromagnetic coupling between the soft layers. The form of the megajoule magnet must, of course, correspond to the optimum operating point on the $\mathrm{BH}$ curve; it should approximate an ellipsoid with $c / a=0.55$.

Equation (1) is based on classical micromagnetic considerations; the sizes of the hard and soft regions must be large compared to atomic dimensions so that a continuum model can be applied. The model must break down when $\lambda_{s}$ or $\lambda_{h}$ is smaller than about $1 \mathrm{~nm}$. Furthermore, the micromagnetic approach takes no account of thermal fluctuations of the free energy, which might be an important source of nucleation in rare-earth magnets at ambient temperature. Fortunately, the nanostructures we have been discussing are very likely to impede domainwall propagation should a reverse nucleus form by thermal nucleation. ${ }^{23}$ Besides, due to the small prefactor $\left(M_{s}-M_{h}\right) / M_{s}, 0.28$, for the $\mathrm{Sm}_{2} \mathrm{Fe}_{17} \mathrm{~N}_{3} / \mathrm{Fe}$ system, the energy product is not much affected if the fraction of the hard phase is increased. We have still an energy product of almost $800 \mathrm{~kJ} / \mathrm{m}^{3}$ (100 MG Oe) in this system when $f_{h}=30 \%$, and we can use the extra hard material to improve the thermal stability and to create pinning centers.

In conclusion, it is likely that substantial improvements in the energy product of permanent magnets can be achieved by exchange hardening in nanoscale combinations of a soft phase and an oriented hard phase, structured according to the principles we have outlined.

This work was supported by the BRITE-EURAM Programme of the European Commision.
${ }^{1}$ Supermagnets, Hard-Magnetic Materials, edited by G. J. Long and F. Grandjean (Kluwer, Dordrecht, 1991).

${ }^{2}$ M. Sagawa, S. Hirosawa, H. Yamamoto, S. Fujimura, and Y. Matsuura, Jpn. J. Appl. Phys. 26, 785 (1987).

${ }^{3}$ J. M. D. Coey and H. Sun, J. Magn. Magn. Mater. 87, L251 (1990); J. M. D. Coey, Phys. Scr. T39, 21 (1991).

${ }^{4}$ Q.-N. Qi, H. Sun, R. Skomski, and J. M. D. Coey, Phys. Rev. 45, 12278 (1992).

${ }^{5}$ R. Coehoorn, D. B. de Mooij, J. P. W. B. Duchateau, and K. H. J. Buschow, J. Phys. (Paris) Colloq. 49, C8-669 (1988).

${ }^{6} \mathrm{~J}$. Ding, P. G. McCormick, and R. Street, J. Magn. Magn. Mater. 124, L1 (1993).

${ }^{7}$ K. -H. Müller, J. Schneider, A. Handstein, D. Eckert, P. Nothnagel, and H. R. Kirchmayr, Mater. Sci. Eng. A133, 151 (1991).

${ }^{8}$ A. Hernando, J. Magn. Magn. Mater. 117, 154 (1992).
${ }^{9}$ E. Kneller and R. Hawig (unpublished); E. Kneller, IEEE Trans. Magn. 27, 3588 (1991).

${ }^{10}$ E. Callen, Y. J. Liu, and J. R. Cullen, Phys. Rev. B 16, 263 (1977).

${ }^{11}$ W. F. Brown, Magnetostatic Principles in Ferromagnetism (Interscience, New York, 1962).

${ }^{12}$ H. Kronmüller, Phys. Status Solidi B 144, 385 (1987).

${ }^{13}$ It can be shown that higher-order anisotropy constants, small fluctuations of $\mathbf{n}$, and magnetostatic effects for macroscopically homogeneous ellipsoids, do not influence the results obtained in the following sections.

${ }^{14}$ R. Skomski, Phys. Status Solidi B 174, K 77 (1992).

${ }^{15}$ D. Givord, Q. Lu, and M. F. Rossignol, in Science and Technology of Nanostructured Magnetic Materials, edited by G. C. Hadjipanayis and G. A. Prinz (Plenum, New York, 1991), p. 635. 
${ }^{16}$ W. F. Brown, Rev. Mod. Phys. 17, 15 (1945). Brown's paradox is that the observed intrinsic coercivity $H_{c}$ is always smaller than the value $H_{c}>2 K_{1} / \mu_{0} M_{0}$ calculated from the theory of nucleation in less finite-size homogeneous ellipsoids. In fact, real systems always exhibit a certain inhomogeneity which is the origin of the much smaller coercivity observed in practice. Here we avoid Brown's paradox by dealing with a system which is inhomogeneous on a scale smaller than $\delta_{h}$, but which contains no micron-size inhomogeneities.

${ }^{17}$ R. Skomski, K.-H. Müller, P. A. P. Wendhausen, and J. M. D. Coey, J. Appl. Phys. 73, 6047 (1993).

${ }^{18}$ S. Nieber and H. Kronmüller, Phys. Status Solidi B 153, 367 (1989); Jifan Hu and H. Kronmüller, Phys. Status Solidi B 172, 67 (1992).

${ }^{19}$ A. Aharoni, Rev. Mod. Phys. 34, 227 (1992).
${ }^{20}$ R. Skomski, Ph.D. thesis, Technische Universität Dresden, 1991.

${ }^{21}$ M. Katter, J. Wecker, C. Kuhrt, L. Schultz, and R. Grössinger, J. Magn. Magn. Mater. 117, 419 (1992).

${ }^{22}$ For $K_{s}<K_{h}$, the small parameter of the perturbation series is $D_{c} / \delta_{h}$, where $D_{c} \approx D$ is the correlation length of the inhomogeneities (cf. Ref. 14). If $D_{c}<\delta_{h}$ the exchange dominates, and the magnetization state is effectively homogeneous.

${ }^{23} \mathrm{~A}$ reversed nucleus will grow spontaneously if its radius exceeds $c \gamma / \mu_{0} M_{0}^{2}$ where $c$ is a constant of order unity and $\gamma$ is the domain-wall energy. If $c=1$ and $\gamma=30 \mathrm{~mJ} / \mathrm{m}^{2}$, the critical radius is $10 \mathrm{~nm}$. However, the thermal energy needed to produce such a nucleus must be at least $4 \pi r^{2} \gamma$, or $4 \times 10^{5}$ $\mathrm{K}$. 